\title{
Confluent 3D-assembly of fibrous structures
}

S. Kazemahvazi ${ }^{1 *}$, N. Khokar ${ }^{2}$, S. Hallstrom ${ }^{3}$, H.N.G. Wadley ${ }^{4}$ and V.S. Deshpande ${ }^{1}$ Affiliations:

${ }^{1}$ Department of Engineering, University of Cambridge, Cambridge, CB2 1PZ, UK.

${ }^{2}$ Biteam AB, SE-167 54, Bromma, Sweden.

${ }^{3}$ KTH, Department of Aeronautical and Vehicle Engineering, SE-100 44, Stockholm, Sweden.

${ }^{4}$ Department of Material Science \& Engineering, School of Engineering and Applied Science, University of Virginia, Charlottesville, VA 22904, USA

\section{*Corresponding author:}

Telephone number: $+46(0) 708830044$

Email address: sohrabk@kth.se

\begin{abstract}
The ability to independently control fiber alignments and structural geometry is critical for design of optimal three-dimensional (3D) fibrous structures. We present a novel method to 3D-assemble carbon fiber structures, containing no seams or adhesive joints, using a confluence of several textile methodologies. A variety of complex structural shapes with tailored fiber topologies are demonstrated to be achievable. These optimized structures are shown to have unprecedented static and dynamic strength as well as damage tolerance and ductility. For example, the energy absorption capacity of a 3D-assembled carbon fiber prismatic sandwich structure is shown to be $300 \%$ greater than a high performance metallic counterpart.
\end{abstract}

Keywords: A. Carbon fibres, A. Sandwich, A. Textile composites, B. Mechanical properties, 3D-weaving 


\section{Introduction}

Tailoring the alignment of fibers in a composite material or structure is crucial for maximizing properties like high strength, stiffness, fracture toughness and damage resilience [1]. Fiber alignment in natural structures often varies with the position to meet local functional objectives [2]. The rachis (shaft) of a bird feather, which is considered to be a highly efficient naturally occurring fibrous structure, can diagrammatically be treated as a beam with webs and faces [3]. The web of the rachis is a layered composite with a $\pm 45^{\circ}$ fiber alignment so as to resist shear forces. It is connected to the faces via a threedimensional (3D) fiber alignment that ensures that the joints have a high toughness and ductility $[3,4]$. Although nature shows many other examples of structures where fiber alignment is analogously optimized, synthetic production of composite structures where fiber alignment and structural topology can be independently tailored in 3D has proved elusive [1].

There are a range of well-established methods to produce two-dimensional (2D) arrangements of reinforcement, e.g. textile technologies using high performance fibers such as carbon fibers [5]. However, structures with a 3D fiber topology are desired due to their superior multi-axial performance [1]. Efforts have been made to modify 2D textile technologies to produce complex 3D shapes using generally so-called 3D-weaving. These have shown promise but are unable decouple macroscopic structural topology and microscopic tow/fiber alignments [6-8]. Most of these 3D solutions are based on the principle of adding out-of-plane reinforcements to a planar 2D weave, examples include z-pinning [9, 10], interlock-weaving [11] and stitching [12, 13]. Well-established 3D textile methods such as braiding $[14,15]$ and knitting [16] have also been demonstrated to directly 
produce near net-shape structures. However, in braiding and knitting the structural geometry and fiber alignments are inherently interlinked, and structures produced by these methods are often sub-optimal. Here, a new method to 3D-assemble continuous long fibers is described that enables independent control over structural geometry and fiber alignment. We can thereby synthetically produce optimal geometries and fiber arrangements for a given function. The method is a confluence of different textile technologies such as weaving and braiding. The innovation lies in circumventing the inherent (physical/geometrical) limitations of the conventional weaving principles [17] in order to realize 3D-assembly of structures.

\section{Materials and manufacturing}

The process to manufacture 3D-assembled carbon fiber structures is briefly described here. It is instructive to compare the performance of the 3D-assembled structure with state-of-the-art material systems currently used in aerospace and marine structures. For this we choose the following two benchmarks: (a) an equal mass and same overall geometry aerospace grade 6061-T6 aluminum alloy beam (denoted 6061-T6) and (b) an equal weight and volume carbon fiber reinforced epoxy (denoted CFRP) sandwich beam with a high performance polymer foam core.

\section{$2.1 \quad 3 D$-assembly of carbon fiber structures}

The 3D-assembled composite beams were manufactured in two steps. First, a dry preform was created using the confluent 3D-assembly process with carbon fiber yarns and then the preform was infused with a polymeric resin using a rigid tool to ensure that the dry preform retained its geometry and fiber alignment under the infusion process. The final as infused 3D-assembled composite beam had an overall geometry and dimensions as depicted in Fig. 1. 


\subsubsection{High-level description of the confluent 3D-assembly process}

The technology for confluent 3D-assembly and the preforms used in this study were supplied by the textile company Biteam $\mathrm{AB}^{1}$. We here provide a high-level overview of the process to highlight the key innovations of the process. The confluent 3D-assembly process involves three innovations to augment the traditional weaving process. First the so-called warp fiber tows are brought in perpendicular to the plane of the weave and the so-called weft tows are inserted at the bend of the warp tows as shown in Fig. 2a to form a 2D-weave. Moreover, the warp tows are brought in at multiple positions along the assembly line in order to produce a multi-layer weave in a single step, see Fig. 2a. In traditional weaving the warp tows are fed in the plane of the weave which physically prevents the single-step production of multi-layer weaves. Another drawback of the inplane warp tow feed is that the feeding machinery occupies space at the inlet of the weave. Since the warp feed machinery in this new process is located above/below the weave we have now opened the possibility to add fabrics that are fed at the inlet of the weave at arbitrary orientations; see Fig. 2b. These "add-on fabrics" can have any desired fiber alignment (Fig. $2 \mathrm{~b}$ shows a $\pm 45^{\circ}$ configuration) and can be produced using a variety of textile techniques (e.g. flat braiding) that are fed into the assembly line. This is the second innovation. The final innovation is that these "add-on fabrics" are interlocked with the weaves via the weft tow insertions as shown in Fig. 2. The tow wavelengths of the weaves and add-on fabrics can be tailored to achieve the desired degree of interlocking. In Fig. 2c an example is shown where the weave and add-on fabric have equal tow wavelengths, which results in a fully interlocked structure. The method is thus a confluence of a range of textile processes which results in 3D

\footnotetext{
${ }^{1}$ Biteam AB, Bromma, Sweden. http://www.biteam.com
} 
interlocked achitectured structures. The method to 3D-assemble architectured structures with webs and faces, inspired the development of a prismatic beam similar to the highly efficient bird feather rachis as shown schematically in Fig. 2d. The 3D-assembled structure comprises two webs that are inclined at approximately $50^{\circ}$ to the faces to create high torsional stiffness and provide lateral stability to the beam. The webs are designed to comprise six laminae, each laminae having a fiber alignment of $\pm 45^{\circ}$ with respect to the beam longitudinal direction. Moreover, the joints of the webs to the faces have a fully interlocked 3D fiber arrangement (Fig. 2c) which maximizes toughness of the joints that are typically the weak links in traditional composite structures. An optical photograph and an $X$-ray computed tomography $(X-\mathrm{CT})$ of the 3D-assembled beam infused with a polymer matrix is shown in Fig. 3a. Additional examples of geometries with varying 3D fiber alignments are shown in Figs. 3b-c.

\subsubsection{Manufacture of the 3D-assembled beams}

All preforms were 3D-assembled via the confluent 3D assembly process as described above using a commercial grade $6 \mathrm{~K}$ tow carbon fiber supplied by TohoTenax, STS-40 [18]. The materials, fiber weights and architecture are specified in Table 1. A photograph of the dry 3D-assembled preform is shown in Fig. 4a and clearly reveals the different fiber alignments in the webs and faces as well as the tight interlocked structure of the weaves which enables the preform to hold its overall structural shape before infusion. The 3D-assembled fiber preforms were infused using aluminum tooling to form an infusion cavity. The tool cavity was designed so that the thickness of the face sheets are $t_{f}=1 \mathrm{~mm}$ and the webs are $t_{w}=2.4 \mathrm{~mm}$. This implied fiber volume fractions of approximately $50 \%$ in the face sheets and $60 \%$ in the webs. An ultra-low viscosity epoxy resin, NM Infusion 664 [19], was infused into the cavity at a pressure of 1 bar. 


\subsubsection{Manufacture of the 6061-T6 aluminum alloy beam}

A high performance metallic reference beam with overall dimensions (i.e. same overall length and thickness) as well as areal weight equal to the 3D-assembled beam was constructed. This beam also had two face sheets and webs inclined at $50^{\circ}$ and was made from $0.8 \mathrm{~mm}$ thick annealed 6061-T6 aluminum (Al) alloy sheets so that the face sheet and web thicknesses were $t_{f}=t_{w}=0.8 \mathrm{~mm}$ (the $\mathrm{Al}$ face sheets were necessarily thinners compared to the 3D-assembled beams so as to achieve the same overall areal mass of the beams). The $\mathrm{Al}$ sheets were annealed in order to improve the formability of the sheets. This allowed the sheets to be CNC-folded into the inclined web structure (referred to subsequently as the corrugated core). Small flats are designed at the base and top of the corrugated core to allow for a strong bonding with the faces. This corrugated core was then metallurgically bonded to $0.8 \mathrm{~mm}$ thick face sheets using dip brazing (an aluminum alloy 4047 braze in foil form). The brazed panel was then subjected to a full solution heat treatment with a water quench and subsequent precipitation hardening to the T6 condition. Dog bone specimens were cut-out from the fabricated sandwich panel in order to confirm that a T6 condition had been reached. The measured yield strength of the Al alloy in this as-fabricated beam was $290 \mathrm{MPa}$ with an ultimate tensile strength of $360 \mathrm{MPa}$ attained at a tensile strain of approximately $10 \%$.

\subsubsection{Manufacture of carbon fiber reinforced epoxy sandwich beam with a foam core} Carbon fiber reinforced plastic (CFRP) sandwich beams were manufactured to provide a reference composite beam to compare with the 3D-assembled beam. The CFRP sandwich beam comprised $1.2 \mathrm{~mm}$ CFRP face sheets (approximately equal to those of the 3D-assembled structure) that were bonded to a PVC foam core. Each face sheet was made from four layers of a $6 \mathrm{k}$ commercial grade carbon fiber plain weave fabric (375 
$\mathrm{gm}^{-2}$ per layer) while the PVC foam core was a high performance closed cell H-grade Divinycell [20] foam with a density of $250 \mathrm{kgm}^{-3}$. The foam core was first sandwiched between the dry carbon fiber fabrics (with warps aligned with the longitudinal beam direction) and the entire assembly was then infused with NM Infusion 664 epoxy (i.e. same matrix as used on the 3D-assembled beam). The asmanufactured CFRP sandwich beam had an areal mass of $8.5 \mathrm{kgm}^{-2}$ and overall thickness of $20 \mathrm{~mm}$. Thus, this beam too had overall dimensions and weight approximately equal to the 3D-assembled beam although it did not have the same structural geometry as it had a foam core rather than a core with webs. Composite sandwich construction with carbon fiber faces and a PVC foam core is used in highperformance naval applications [21] and thus serves as a useful benchmark.

\subsection{Experimental protocol}

The mechanical performance of the 3D-assembled beam and its benchmarks are investigated through two types of experiments; (i) a quasi-static 3-point bend tests and (ii) a set of high velocity soft impact experiments that mimic a bird strike [22].

\subsubsection{Quasi-static 3-point bend test and X-CT scanning}

The set-up sketched in Fig. 5a was used to measure the flexural response of all three beam types. Beams of overall length $300 \mathrm{~mm}$ were simply supported so that a test span of $L=150 \mathrm{~mm}$ was obtained. The supports were $25 \mathrm{~mm}$ wide flat bars to avoid local indentation failure and these bars were supported on rollers that allow free rotation of the beams (Fig. 5a). The central load was also applied via a bar of the same size as shown in Fig. 5a. The displacement $\delta$ of the central indenter was measured via a laser extensometer. Photographs were taken at regular intervals during the bend tests to image the beam throughout the entire deformation history. The central indenter was 
displaced at a rate of $1 \mathrm{~mm}$ per minute via a screw driven test machine and the applied load $P$ measured via the load cell of the test machine. In addition to the standard 3-point bend setup, an experiment was performed on the 3D-assembled beam where the test was interrupted at specified central indenter displacements in order to perform a full 3D $X$ CT scan of the beam. Using the data from the $X$-CT scans, the progression of damage throughout the bending test as shown in the main text, was visualized. At-least 5 different samples of the 3D-assembles beams were tested and these tests: minimal scatter (less than 10\%) in the measured load versus displacement response was observed confirming the fidelity of the manufacturing process.

\subsubsection{Impact loading test}

The beams were impacted at mid-span by Duocell aluminum foam [23] projectiles of density $\rho=300 \mathrm{kgm}^{-3}$, length $l_{0}=50 \mathrm{~mm}$ and diameter $d=50.6 \mathrm{~mm}$. The beams of length $300 \mathrm{~mm}$ were simply supported on rollers of diameter $25 \mathrm{~mm}$ such that the test span of the beams was $L=150 \mathrm{~mm}$ as sketched in Fig. 5b. The foam slugs were fired at a velocity $v_{0}$ in the range $100-180 \mathrm{~ms}^{-1}$ from a gas gun of bore $50.8 \mathrm{~mm}$ and barrel length $4.5 \mathrm{~m}$. The velocity of the projectile was measured at the exit of the barrel using laser-velocity gates. The dynamic transverse deformation of the beams and beam midspan displacements were observed using a Phantom v.1600.1 high-speed camera taking images with an inter-frame time and an exposure time of $20 \mu$ s and $0.4 \mu \mathrm{s}$, respectively.

\section{Results and discussion}

\subsection{Quasi-static mechanical performance}

The load $P$ versus mid-span deflection $\delta$ in the quasi-static 3-point bend test is plotted in Fig. 6a for the three beam types. The 3D-assembled beam has a similar flexural stiffness 
as the 6061-T6 beam but outperforms the CFRP and 6061-T6 beams in flexural strength and especially energy absorption (see Fig. 6b). The tests reveal a very high energy absorption capability for the 3D-assembled beam, which is more than $300 \%$ higher than for the 6061-T6 beam and $40 \%$ higher than for the CFRP beam (energy absorption calculated up to $\delta=20 \mathrm{~mm}$ ). The energy absorption of the 6061-T6 beam and the CFRP beam are linked to the high tensile ductility of the aluminum alloy and the high shear ductility of the polymer foam [20], respectively. However, the 3D-assembled beam is made exclusively from brittle materials; carbon fibers and an epoxy matrix. The ductility of the 3D-assembled beam originates from the optimized fiber architecture that promotes progressive crushing of carbon fiber tows within the joints rather than localized damage. The evolution of progressive damage during the flexural test is visualized through $X$-CT scans at regular intervals during the test; see Fig. 6c. Immediately after the peak load, compressive failure of the core webs is observed together with progressive micro-buckling of the fibers in the top face sheet and in the top joint as shown in the cross-section and top joint view in Fig. $6 \mathrm{c}(\delta=3 \mathrm{~mm})$. The exceptional energy absorbing capability of the 3D-assembled beam is a result of this pseudo-ductility. Due to the high toughness of the 3D-assembled joints, the beam is also able to carry significant load post peak load as shown in Fig. 6a.

\subsubsection{Failure mechanisms of the beams under static three-point bend loading}

A montage of photographs showing the deformation of the beams subject to quasi-static three-point bending is included in Fig. 7 with the corresponding load $P$ versus displacement $\delta$ curves plotted in Fig. 6a. The deformation/failure mechanisms for each of the three beam types are discussed in turn. 
Consider first the 6061-T6 beam. The peak load occurs at an indenter displacement of $\delta=1.1 \mathrm{~mm}$. This peak load is set by the plastic wrinkling of the top face sheet which results in a decaying load. Subsequently, the webs of the core plastically buckle and the load begins to level out around $1.6 \mathrm{kN}$. Continued deformation occurs by a combination of core indentation and global bending until the core is fully densified at approximately $\delta=20 \mathrm{~mm}$ at which point indentation ceases.

The $P$ versus $\delta$ curve in Fig. 6a clearly shows that the CFRP beam deforms elastically until $\delta \approx 3 \mathrm{~mm}$. Subsequently, the deformation is primarily by plastic shearing of the foam core as seen in the montage in Fig. 7. The top face sheet undergoes microbuckling failure immediately underneath the central indenter at $\delta \approx 6 \mathrm{~mm}$ which results in a sharp drop in the applied load. Subsequently, the top face sheet carries nearly no load and the beam deforms by crushing of the foam core with the neutral axis near the bottom face sheet of the beam. This implies that the tensile strains induced in the bottom face sheet remain small and the tensile strains in the foam are also negligible. The observed ductile beam response is a result of the compressive ductility of the foam. The deformation sequence of the 3D-assembled beam is similar in many respects to the CFRP beam. After an initial elastic deformation phase, the top face sheet buckles under the central indenter and fails at a displacement $\delta \approx 2.4 \mathrm{~mm}$ (and applied load $P \approx 10 \mathrm{kN}$ ). Subsequently, the beam deforms by progressive longitudinal crushing of the joint between the core webs and the top face sheet combined with the longitudinal compression of the core webs as discussed previously and seen in the $X$-CT scans in Fig. 6c. We emphasize here that unlike the more conventional adhesively jointed composite corrugated core structures [24], here the joint crushes but does not result in debonding between the face sheet and core members despite a rather small joint area. 
This progressive crushing occurs at a relatively low stress and causes a shift of the neutral axis of the beam towards the bottom face sheet. This again implies that the bottom face sheet is subjected to relatively low tensile strains despite the large bending displacement $\delta$ that the beam is subject to. Thus, similar to the CFRP beam the ductile response of the 3D-assembled beam is due to the neutral axis of the beam being located near the bottom face sheet during the plateau phase of the $P$ versus $\delta$ curve. However, the deformation mode of the 3D-assembled beam differs in three critical respects from the CFRP beam: (i) the core is stiff and strong in shear and hence the beam deforms in a global bending mode rather than by core shear even during the initial stages of the deformation; (ii) the core webs prevent macro-buckling and/or separation of the joint between the webs and the top face sheet and efficient load carrying capacity is thus maintained; and (iii) the core webs crush in a relatively brittle manner unlike the ductile compression of the foam core in the CFRP beam. The main reason that the plateau load of the 3D-assembled beam is higher compared to that of the CFRP beam is that the separation between the top joint and the bottom face sheet is maintained which sustains the sandwich effect even at large deflections.

The measured stiffness and peak strengths of all the 3 beam types are in excellent agreement with well-established formulae for these types of sandwich structures; see Ref. [25] and not repeated here for the sake of brevity. However, these analyses (and also finite element analysis) of the 3D-assembled beams using standard composite models such as the Hashin model [26] predict catastrophic failure of the 3D-assembled beams immediately after peak load. This is not observed in the measurements reported here. The development of models for 3D-assembled structures with integrally woven joints is suggested as a topic for future studies. 


\subsection{Dynamic mechanical performance}

The temporal variation of the mid-span deflection $\delta^{b}$ of the non-impacted face (rear face) is plotted in Fig. 8a for impact at an initial projectile velocity $v_{0}=130 \mathrm{~ms}^{-1}$ (see Fig. 9 for the corresponding sequence of high-speed photographic images). The 3Dassembled and CFRP beams arrest the projectiles and have a well-defined maximum deflection $\delta_{\max }^{b}$ but the 6061-T6 beam undergoes excessive deformation and is pushed through the supports. Post-impact photographs of the three beam types are presented in Fig. $8 \mathrm{~b}$. At $v_{0}=130 \mathrm{~ms}^{-1}$, slight damage to the web is observed for the $3 \mathrm{D}$-assembled beam as shown in the $X$-CT scan in Fig. 8c. However, it is clear from Fig. $8 \mathrm{~b}$ that the 6061-T6 beam has failed at the same impact velocity due to excessive deformation while the CFRP beam has cracked through the core. The good performance of the 3Dassembled beams is further exemplified in the plot of $\delta_{\max }^{b}$ versus $v_{0}$ in Fig. 8d. At $v_{0}=100 \mathrm{~ms}^{-1}$, all three beams show minimal damage but even then the 6061-T6 and CFRP beams undergo approximately 10 and 3 times higher deflection, respectively, compared to the 3D-assembled beam. The amount/effect of impact damage is quantified by performing quasi-static 3-point bend tests (similar to those described above) on the beams impacted by the $\mathrm{Al}$ foam at $v_{0}=100 \mathrm{~ms}^{-1}$ and $130 \mathrm{~ms}^{-1}$. The post impact residual properties are measured and included in Fig. 6b, showing that the 3D-assembled beam retains a larger fraction of all its measured pre-impact properties compared to the CFRP and 6061-T6 beams. Hence, 3D-assembled beams not only undergo smaller deflections at low impact velocities, but also withstand higher impact velocities without failure. 


\subsubsection{Failure mechanisms in the impact loading tests}

A montage of high-speed photographs taken during the $130 \mathrm{~ms}^{-1}$ impact tests on the three types of beams is included in Fig. 9. Here we discuss the deformation mechanisms inferred from these photographs for the three beam types.

Consider first the 6061-T6 beam. Early in the deformation history, the impact results in plastic buckling of the webs of the core immediately under the impact site and associated deformation of the impacted face sheet. However, in the early stages of the deformation there is nearly no deformation of the rear face sheet and the core webs exert low transverse pressure on the rear face sheet. As the core densifies, the stress it exerts on the rear face sheet increases and forces it to deflect, and the beam enters a global bending mode when the velocities of the front and rear face sheets equalize as discussed in [27]. No tearing of the Al sheets in the core or face sheets is observed but the complete loss in residual strength of the beam (Fig. 6b) is a result of the large beam deflections and core indentation.

By contrast the CFRP beam shows little core indentation upon impact and it is clear from the photos in Fig. 9 that the beam deforms primarily by core shear. The large shear strains in the foam core result in shear fracture of the core near the roller supports. The fracture then initiates de-bonding of the core from the face sheets and results in the loss in residual static strength of the CFRP beam seen in Fig. $6 b$.

While the geometry of the 3D-assembled beam is similar to the 6061-T6 beam the deformation mode is markedly different. The high strength and stiffness of the core members imply that upon impact of the foam projectile, the core initially undergoes negligible deformation but the unsupported parts of the impacted face sheet acquire a high velocity. This results in a large deformation and fracture of the front face sheet 
under the impact site although the junction between the face sheet and the core remains relatively intact. In this initial stage, the rear face sheet of the beam has undergone negligible deformation. Subsequently, the beam deforms by the longitudinal compression of the joint of the front face sheet and the adjacent parts of the webs of the core. Consistent with the observations under static loading discussed above, the 3Darrangement of fibers in the joint results in a pseudo-ductile compressive response of the joint and the core at a relatively low value of compressive strength. The neutral axis of the beam during subsequent deformation is thus close to the rear face sheet that is under tension. However, the low location of the neutral axis implies that the tensile strain in the rear face sheet remains subcritical and thus the beam continues to deform and absorb energy by the compression of the joint between the front face sheet and core as well as of the core webs. The relatively high residual static strength of the beam comes from the fact that the rear face sheet remains intact, the joint between the front face and the core webs is prevented to disintegrate and that the core webs do not buckle. This maintains the separation of the rear face sheet and the joint between the core and the front face sheet which implies that the beam retains a large fraction of the "sandwich effect" that endows it with its high bending stiffness and strength.

\section{Concluding remarks}

The ability to control the alignment of high performance fibers in 3D seamless structures with complex geometries and optimized/tailored fiber topologies is shown to result in structures with excellent mechanical properties. The manufacturing technique is based on a confluence of textile technologies implying that it is readily scalable to construct larger (or smaller) structures. We thus suggest that the opportunity now exists 
to construct a new generation of damage resilient high performance composites for a range of applications such as aircraft structures, automobiles and bio-medical implants.

\section{Acknowledgements}

We are grateful to the Defense Advanced Research Projects Agency (Grant Number W91CRB-10-1-005) for the financial support of this research. We also acknowledge F.

Winberg, B. Starosta, A. Heaver, K. Dharmasena, D. Glover and S. Marshall for support. We are grateful for the materials that have been supplied by DIAB and Nils Malmgren AB.

\section{References}

[1].Fratzl P. (2012) A Composite Matter of Alignment. Science, 335, 177-178.

[2].Fratzl P., (2007) Nature's hierarchical materials, Prog. Mater. Sci., 52, 1263-1334.

[3].Lingham-Soliar T. (2014) Feather structure, biomechanics and biomimetics: the incredible lightness of being, J Ornithol, 155, 323-336.

[4].Laurent C.M. et al. (2014) Nanomechanical properties of bird feather rachises. J. R. Soc.Interface 1:20140961.

[5].TW.Chou, Microstructural Design of Fiber Composites (Camb. Univ. Press, 1992).

[6].Mouritz A.P., et al. (1999) Review of applications for advanced three-dimensional fibre textile composites, Comp. Part A Appl. Sci. Manuf., 30, 1445-1461.

[7].Rudov-Clarka S., et al. (2003) Fibre damage in the manufacture of advanced threedimensional woven composites, Comp. Part A Appl. Sci. Manuf., 34, 963-970.

[8].Lee L., et al. (2002) Effect of weaving damage on the tensile properties of threedimensional woven composites Comp. Struct., 57, 405-413, (2002).

[9].Freitas G, Magee C, Dardzinski P, Fusco T. (1994) Fiber insertion process for improved damage tolerance in aircraft laminates. J Adv Mater, 25, 36-43 
[10]. A. P. Mouritz (2007) Review of z-pinned composite laminates, Comp. Part A Appl. Sci. Manuf . 38, 2383-2397.

[11]. Ivanov D. S. et al. (2009) A comparative study of tensile properties of noncrimp 3D orthogonal weave and multi-layer plain weave e-glass composites. Comp. Part A Appl. Sci. Manuf., 40, 1144-1157.

[12]. Malcom, A.J., et al. (2013) Compressive response of glass fiber composite sandwich structures, Comp. Part A Appl. Sci. Manuf., 54, 88-97.

[13]. George, T., et al. (2014) Hybrid core carbon fiber composite sandwich panels: Fabrication and mechanical response, Comp. Struct., 108, 696-710.

[14]. Dian-sen Li, et al. (2009) Microstructure and mechanical properties of 3D- fivedirectional braided composites, Int. J. Solid. Struct., 46, 3422-3432.

[15]. Potluri P. et al. (2003) Geometrical modelling and control of a triaxial braiding machine for producing 3D preforms, Comp. Part A Appl. Sci. Manuf., 34, 481-492.

[16]. Kamiya R., et al. (2000) Some recent advances in the fabrication and design of three-dimensional textile preforms: a review Comp. Sci. Tech., 60, 33-47.

[17]. P. R. Lord, M. H. Mohamed, Weaving: Conversion of Yarn to Fabric, Merrow Publishing Co. Ltd., Herts, UK, (1973).

[18]. TohoTenax; STS40 datasheet available at http://www.tohotenax-eu.com.

[19]. Nils Malmgren AB; NM infusion 664 datasheet

[20]. DIAB Group; Divinycell H250 datasheet

[21]. Zenkert D. et al. (2005) Damage tolerance assessment of composite sandwich panels with localised damage Comp. Sci. Tech., 65, 2597-2611.

[22]. Heimbs S., Bergmann T. (2012) High-Velocity Impact Behaviour of Prestressed Composite Plates under Bird Strike Loading, Int. J. Aerospace. Eng.

[23]. ERG Aerospace Corporation, http://www.ergaerospace.com/.

[24]. Zang J, Supernak P, Mueller-Alander S, Wang C H. Improving the bending strength and energy absorbtion of corrugated sandwich composite structures. Materials and Design.2013;52:767-773. 
[25]. Zenkert D, An introduction to sandwich construction. EMAS. 1997

[26]. Hashin Z. Failure criteria for unidirectional fiber composites. J Appl Mech 1980;47:329-34.

[27]. Tilbrook M.T., Deshpande V.S., Fleck N.A. (2006) The impulsive response of sandwich beams: Analytical and numerical investigation of regimes of behaviour, J. Mech. Phy. Solids, 54, 2242-228 


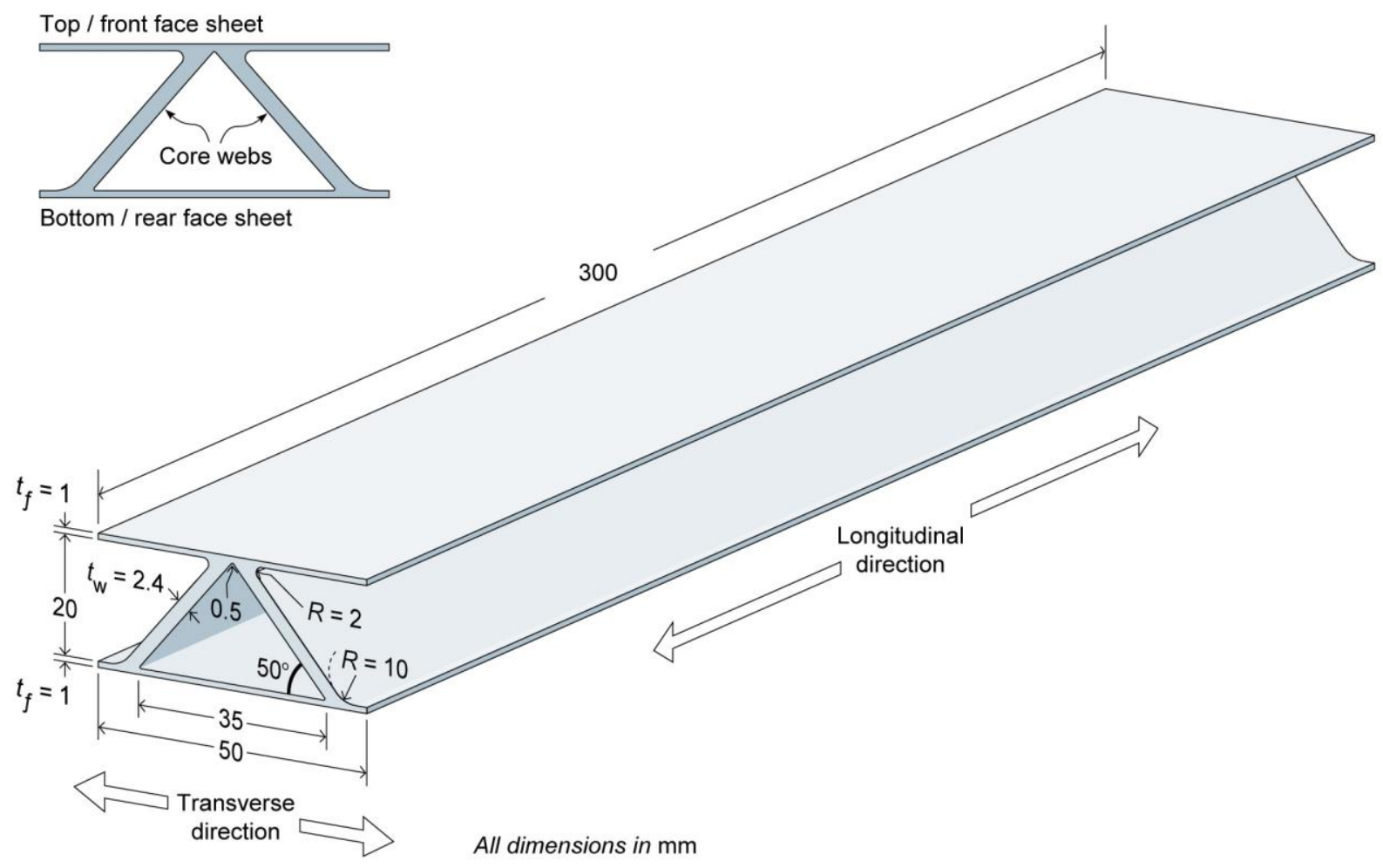

Fig. 1. Sketch showing the overall geometry of the 3D-assembled bird feather rachis-like beam. The naming conventions for the different parts and directions of the beam are also indicated. 


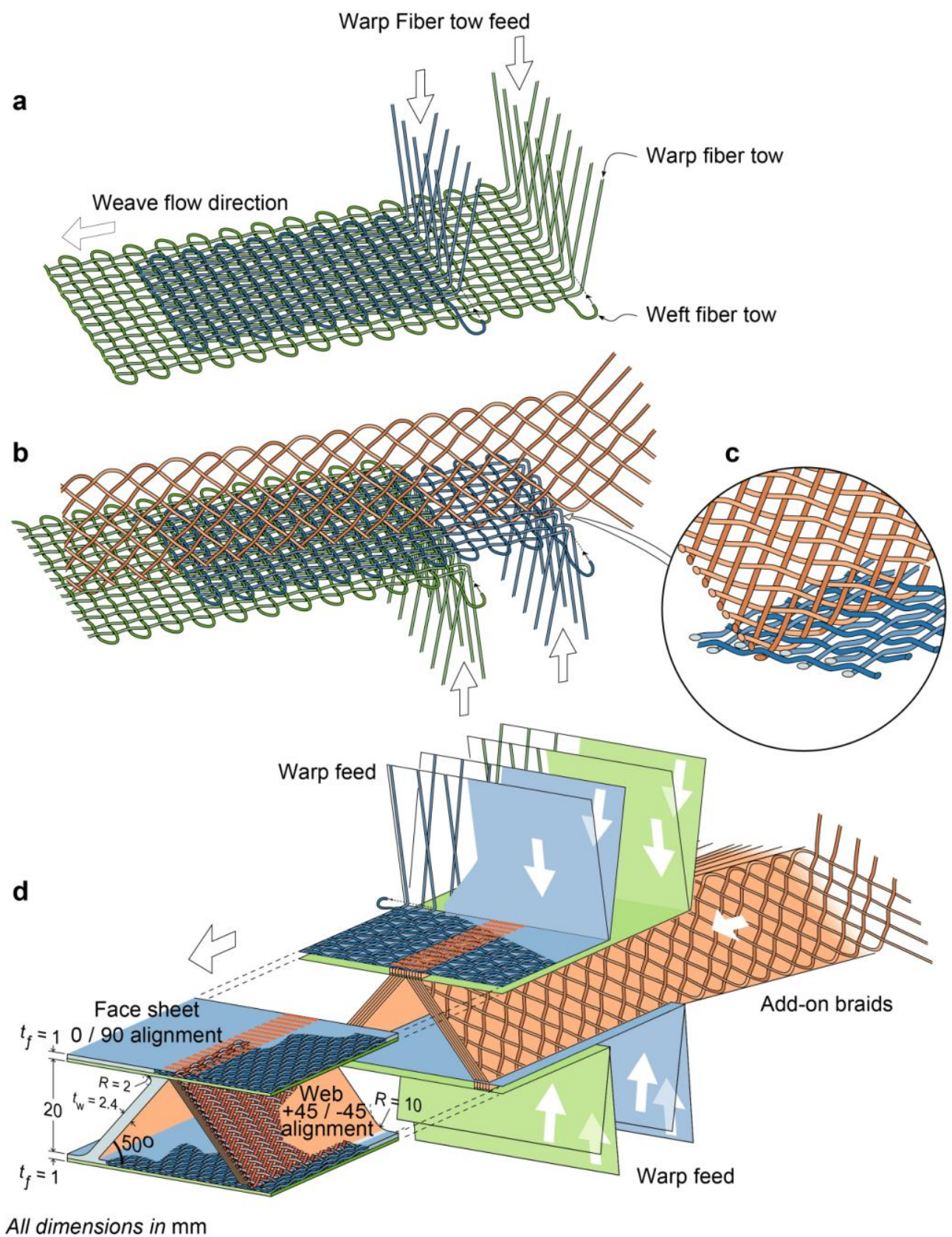

Fig. 2. Confluent 3D-assembly of fibrous structures. To enhance visual clarity, all fabrics are deliberately shown much more "open" than actually produced. (a) The multiple out-of-plane warp fiber tow feeds showing how several layers of woven fabric can be produced in one step. (b) $3 \mathrm{D}$-assembly of a T-section beam with $\pm 45^{\circ}$ fiber alignment in the web (here shown as a flat braid) and $0 / 90^{\circ}$ fiber alignment in the flange. (c) Detailed view of the joint between the web and flange in the T-section beam showing the interlocking of the fibers. (d) Schematic view of a confluent 3D-assembled sandwich beam structure. The tailored fiber alignments were realized by a combination of out-of-plane weaving of the face sheets and flat braiding of the webs. 

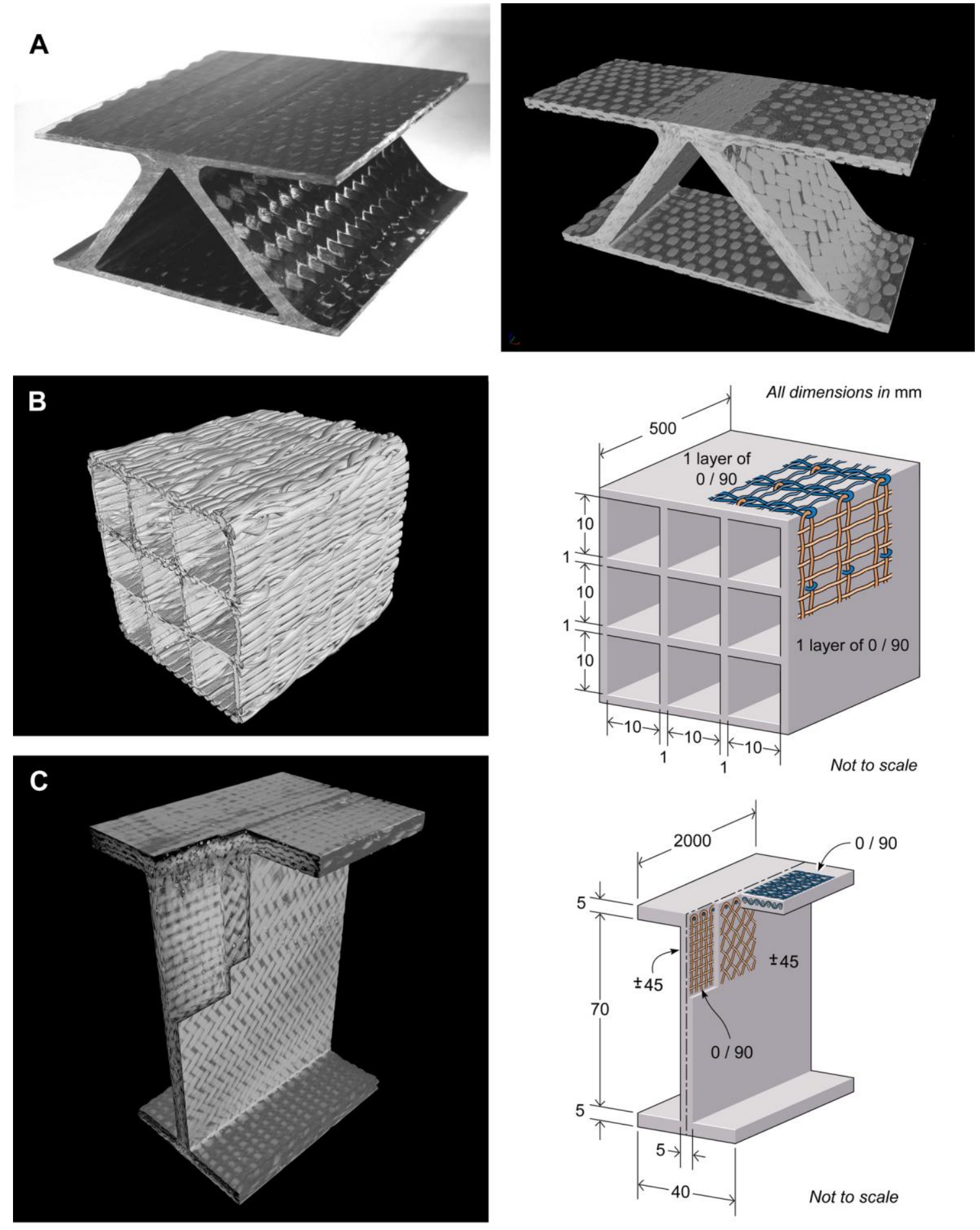

Fig. 3. A variety of structures have been produced using fiber $3 \mathrm{D}$-assembly processes. (a) A $50 \mathrm{~mm}$ long section of a 3D-assembled and infused carbon fiber bird feather rachis-like beam (photograph to the left and $20 \mathrm{~mm}$ long $X$-CT scan to the right). (b) A section of a 9-cell square honeycomb carbon fiber duct structure ( $X$-CT scan) with interlocking joints and unbalanced $0 / 90^{\circ}$ fiber alignment. (c) Large scale I-beam with a combination of 2 layers of $0 / 90^{\circ}$ and 4 layers of $\pm 45^{\circ}$ fiber alignments in the web interlocked with 4 layers of $0 / 90^{\circ}$ fabrics in the flanges (drawing only shows single layers for graphical clarity). This beam was 3D-assembled into a $2000 \mathrm{~mm}$ long part and demonstrates the scalability of the confluent 3Dassembly method. 

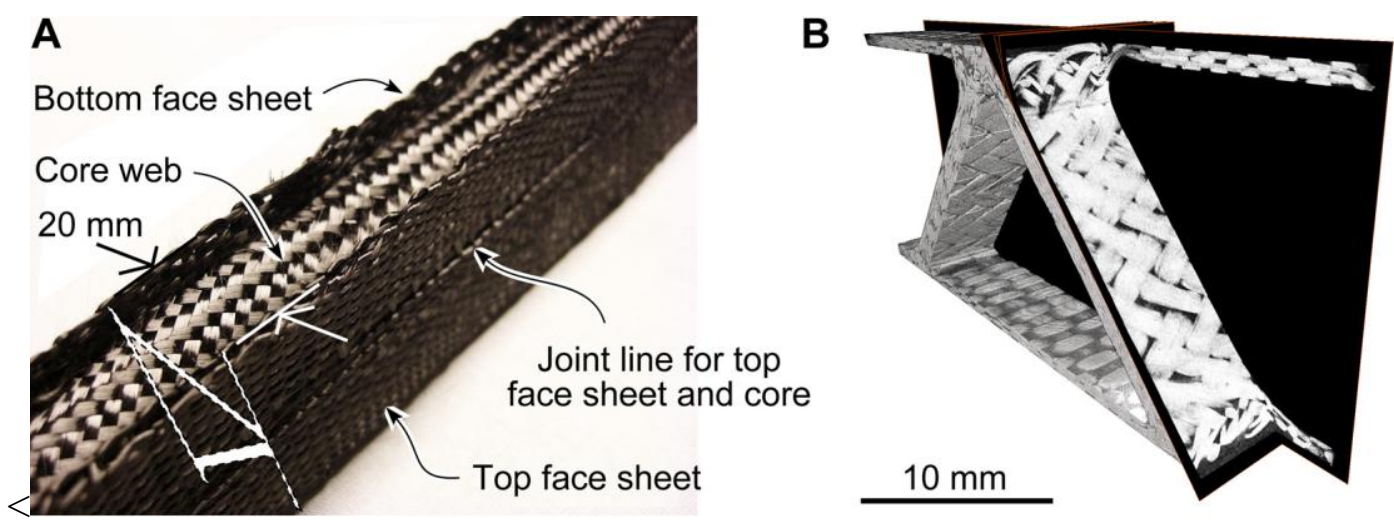

Fig. 4. Images of the fiber alignments in the 3D-assembled bird feather rachis-like beam. (A) Photograph of dry preform. (B) $X$-CT scan section cut showing weave architecture in core webs, face sheets and joints. Scale bar is $10 \mathrm{~mm}$.

A

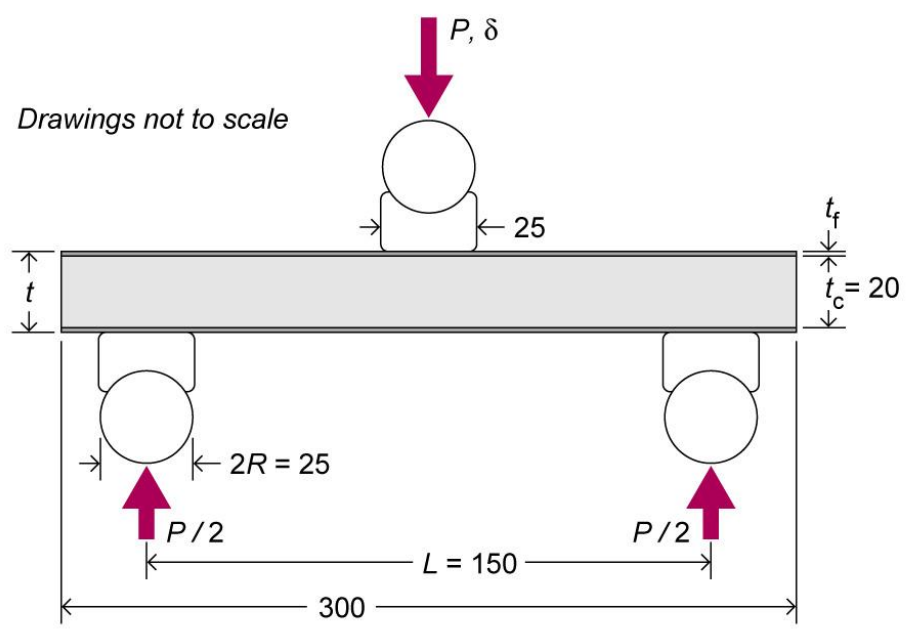

B

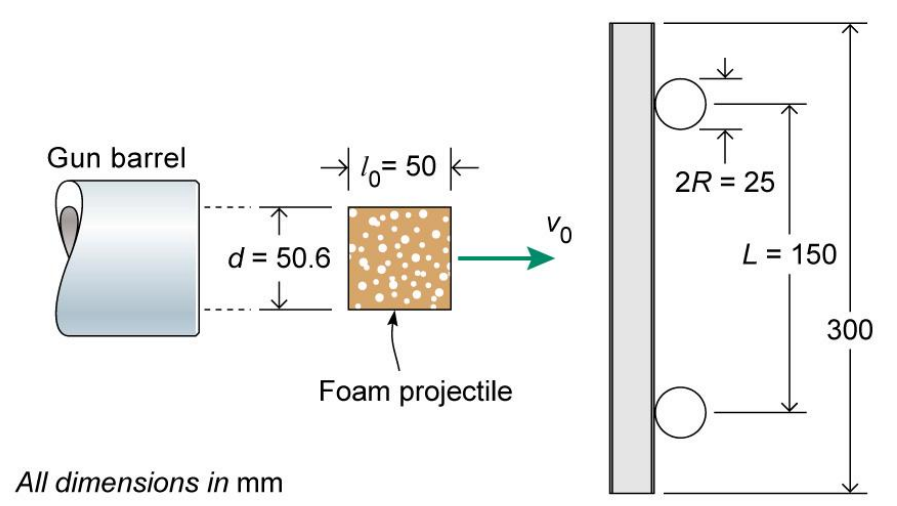

Fig. 5: Sketches of the experimental test setups used for (a) Quasi-static 3-point bending of the beams and (b) foam impact testing of the simply supported beams. 
A

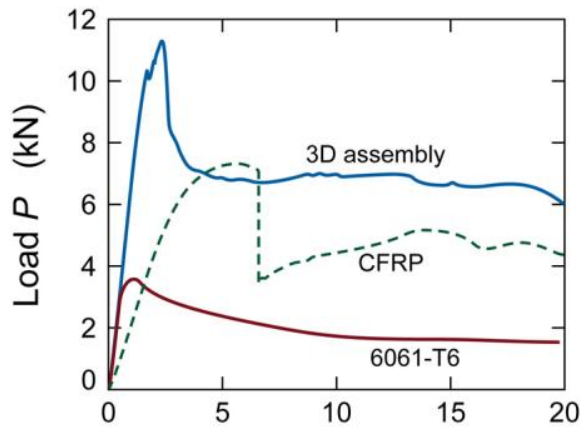

Mid-span displacement $\delta(\mathrm{mm})$
B

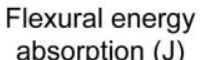

Flexural
stiffness $(\mathrm{kN} / \mathrm{mm})$

Flexural peak load $(\mathrm{kN})$
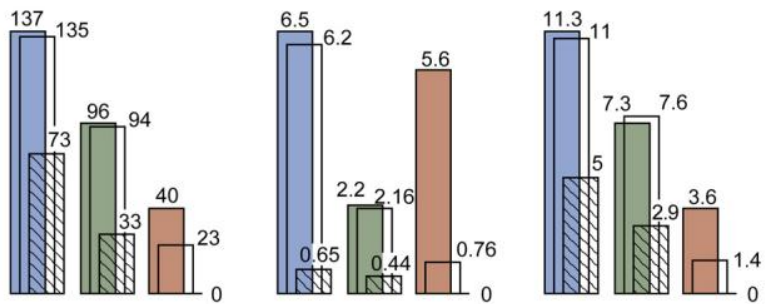

$\square$ 3D assembly $\square$ CFRP $\square$ 6061-T6

$\square$ Post-impact $(100 \mathrm{~m} / \mathrm{s}) \quad \square$ Post-impact $(130 \mathrm{~m} / \mathrm{s})$

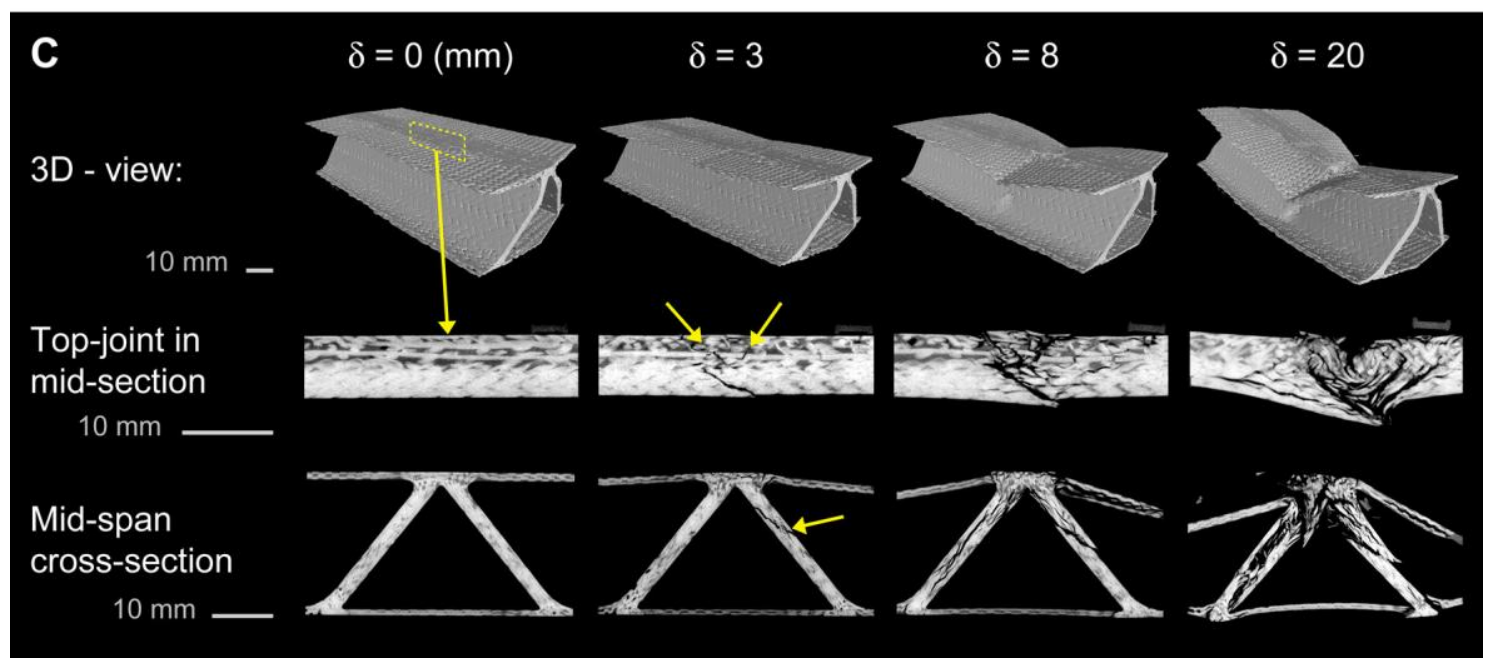

Fig. 6. Quasi-static 3-point bend tests. (a) Load, $P$, versus displacement, $\delta$. (b) Comparisons of flexural energy absorption, stiffness and peak load as well as the corresponding post-softimpact properties. (c) $X$-CT scans of the 3D-assembled sandwich beam performed during the flexural testing. The scans show progressive crushing of the top joint.

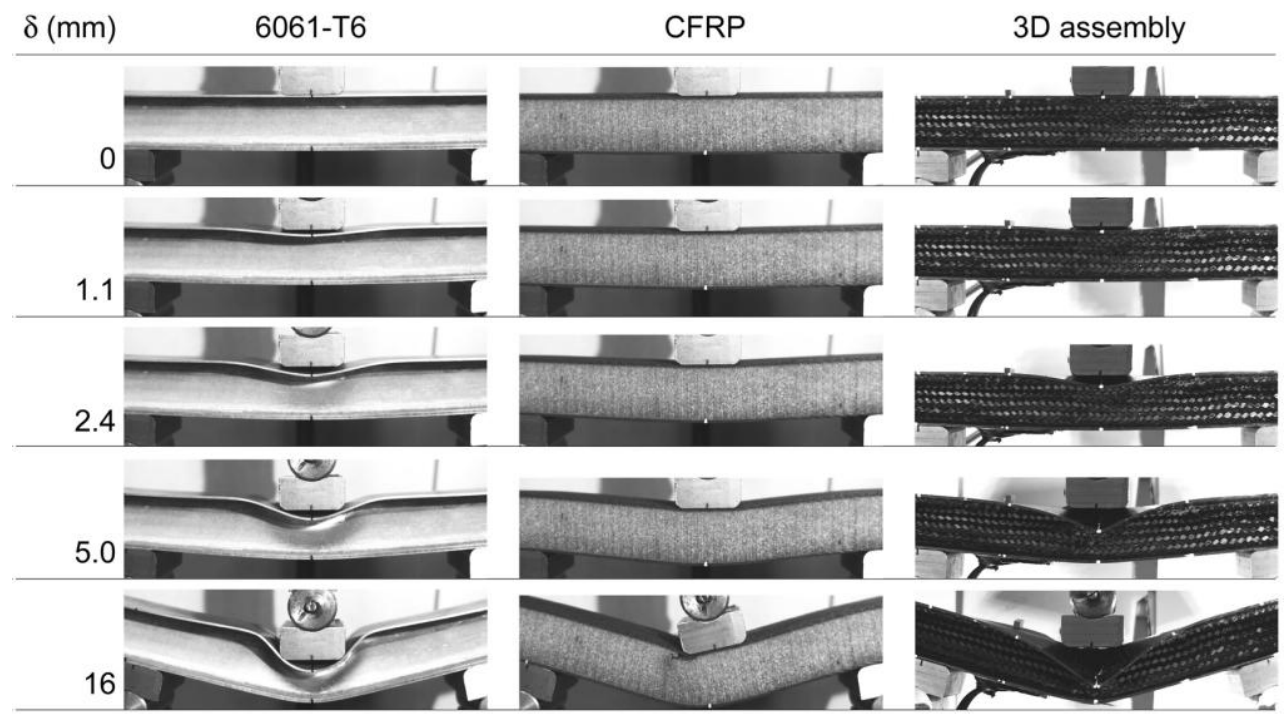

Fig. 7. Montage of photographs showing the deformation of the three beam types under quasi-static three-point bending. 
A
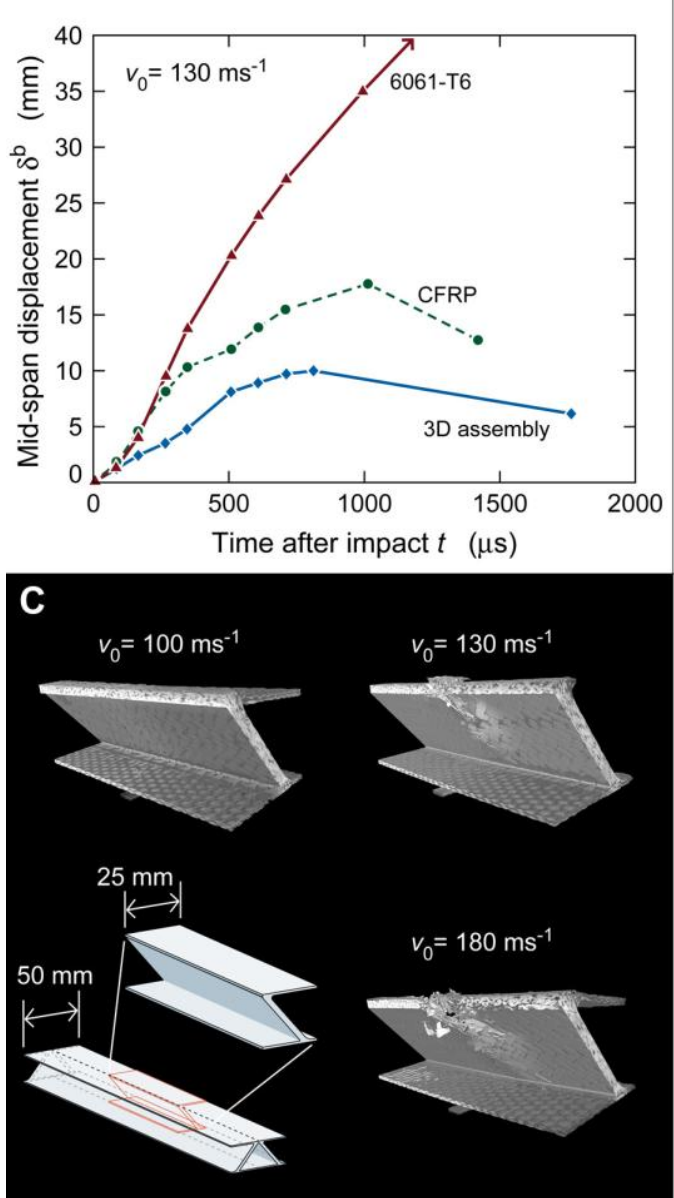

B

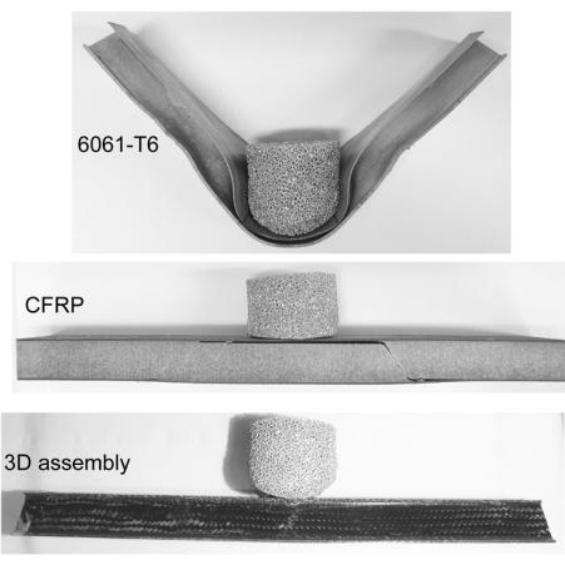

D

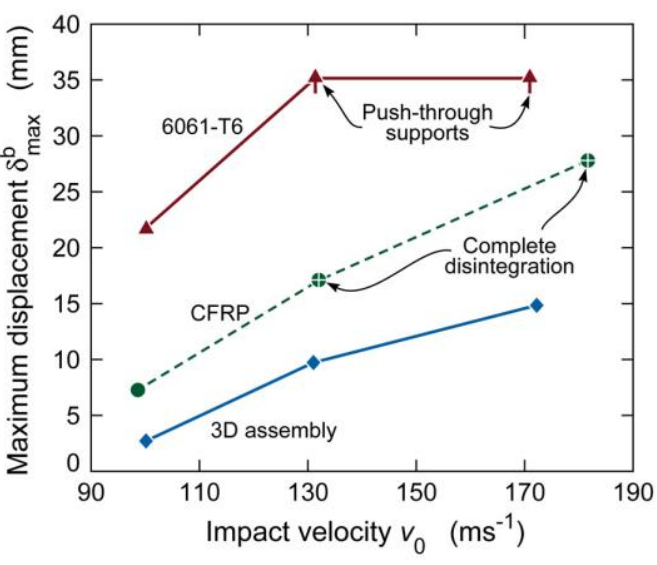

Fig. 8. Impact testing of beams. (a) Mid-span displacement as function of time t for impact at $v_{0}=130 \mathrm{~ms}^{-1}$. Time $\mathrm{t}=0$ corresponds to the instant of impact. (b) Post-impact photographs of the three beams impacted at $v_{0}=130 \mathrm{~ms}^{-1}$. (c) Post-impact $X$-CT scans of a central section of the 3D-assembled beam structure tested at three different impact velocities. A sketch showing the scanned section is included in the bottom left corner. (d) Maximum mid-span displacement as function of impact velocity. 


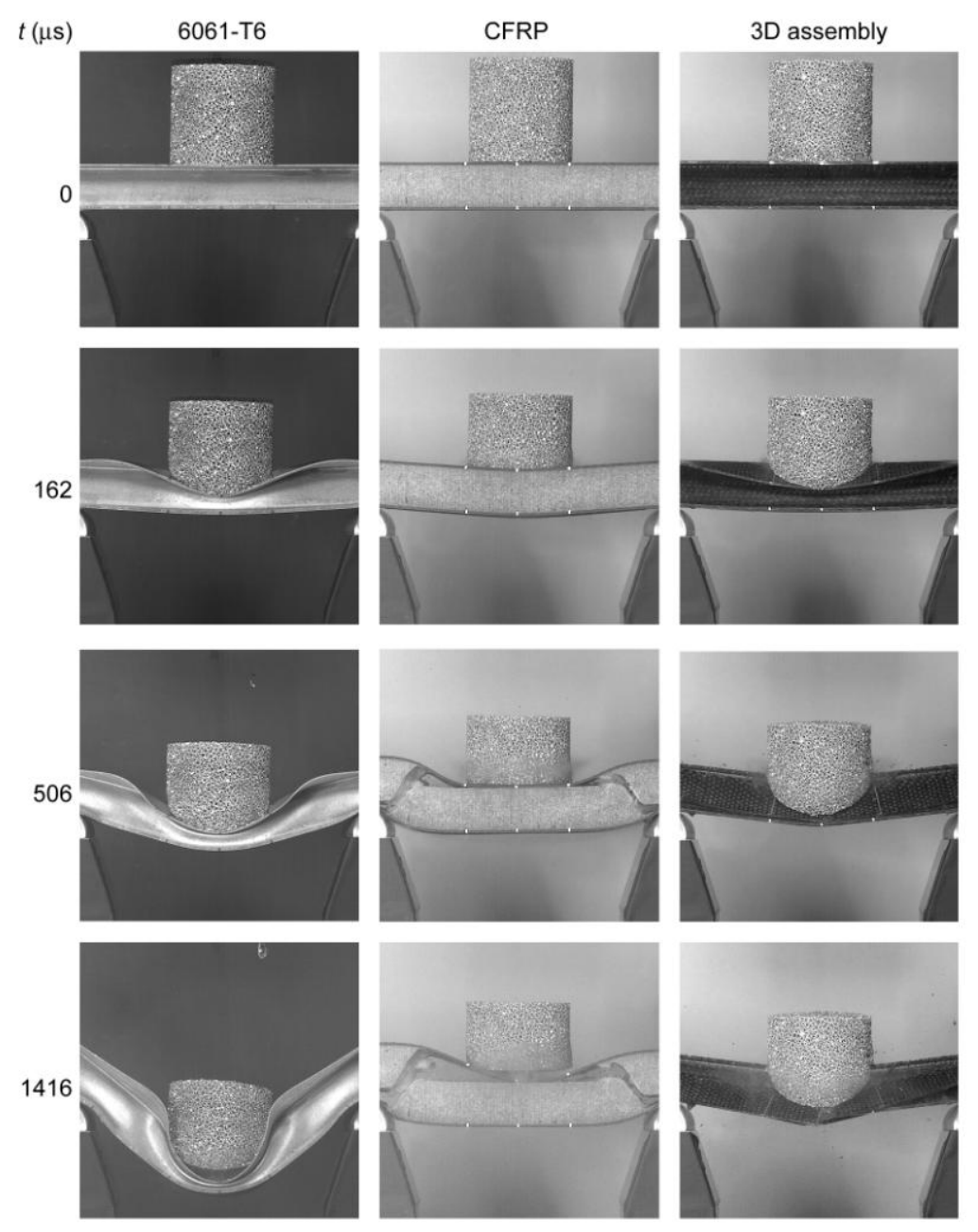

Fig. 9. Montage of high speed photographs showing the deformation of the beams subjected to foam impact at $v_{0}=130 \mathrm{~ms}^{-1}$. Time $t=0$ corresponds to the instant of impact.

\begin{tabular}{lc|c|c}
\hline & $\begin{array}{c}\text { Top face sheet } \\
\text { (2 layers) }\end{array}$ & $\begin{array}{c}\text { Core web } \\
\text { (6 layers) }\end{array}$ & $\begin{array}{c}\text { Bottom face sheet } \\
\text { (2 layers) }\end{array}$ \\
\hline Fiber type & \multicolumn{2}{c}{ TohoTenax, STS40 carbon fibre, 6k/tow } \\
\hline Matrix type & \multicolumn{3}{c}{ NM Epoxy Infusion 664} \\
\hline $\begin{array}{l}\text { Fibers in longitudinal } \\
\text { direction }\end{array}$ & $524 \mathrm{gm}^{-2}$ & $0 \mathrm{gm}^{-2}$ & $524 \mathrm{gm}^{-2}$ \\
\hline $\begin{array}{l}\text { Fibers in transverse } \\
\text { direction }\end{array}$ & $360 \mathrm{gm}^{-2}$ & $0 \mathrm{gm}^{-2}$ & $360 \mathrm{gm}^{-2}$ \\
\hline $\begin{array}{l}\text { Fibers at } \mathbf{+ 4 5} \text { degree to } \\
\text { the longitudinal direction }\end{array}$ & $0 \mathrm{gm}^{-2}$ & $2496 \mathrm{gm}^{-2}$ & $0 \mathrm{gm}^{-2}$ \\
\hline $\begin{array}{l}\text { Areal weight of dry } \\
\text { preform }\end{array}$ & $4.7 \mathrm{kgm}^{-2}$ \\
\hline $\begin{array}{l}\text { Areal weight of infused } \\
\text { composite beam }\end{array}$ & $8.0 \mathrm{kgm}^{-2}$ \\
\hline
\end{tabular}

Table 1: Specification of the materials and fiber weights in the 3D-assembled sandwich beam. 